Proceedings

\title{
Folic Acid-Targeted Doxorubicin Drug Delivery System for Triple-Negative Breast Cancer Treatment ${ }^{+}$
}

\author{
Eleni Lamprou 1,2, Spyridon Mourtas 1,2, Maria Mantzari 1,2, Antonia Marazioti 1, Foteini Gkartziou 1,2 and \\ Sophia G. Antimisiaris 1,2,*
}

Citation: Lamprou, E.; Mourtas, S.;

Mantzari, M.; Maraziotis, A.;

Gkartziou, F.; Antimisiaris, S.G.

Folic Acid-Targeted Doxorubicin

Drug Delivery System for

Triple-Negative Breast Cancer

Treatment. Proceedings 2021, 78, 4.

https://doi.org/10.3390/IECP2020-086

60

Published: 1 December 2020

Publisher's Note: MDPI stays neutral with regard to jurisdictional

claims in published maps and institutional affiliations.

Copyright: (C) 2020 by the authors. Licensee MDPI, Basel, Switzerland. This article is an open access article distributed under the terms and conditions of the Creative Commons Attribution (CC BY) license (http://creativecommons.org/licenses /by/4.0/).
1 Laboratory of Pharmaceutical Technology, Department of Pharmacy, University of Patras, 26504 Patras, Greece; eleni_lamprou@upatras.gr (E.L.); s.mourtas@upatras.gr (S.M.); maria.mantzari1@gmail.com (M.M.); amarazioti@upatras.gr (A.M.); fotini_gartz@yahoo.gr (F.G.)

2 Institute of Chemical Engineering \& Sciences, FORTH/ICES, 26504 Patras, Greece

* Correspondence: santimis@upatras.gr; Tel.: +30-2610-962-332

† Presented at the 1st International Electronic Conference on Pharmaceutics, 1-15 December 2020; Available online: https://iecp2020.sciforum.net/.

\begin{abstract}
Triple-negative breast cancer (TNBC) is a highly aggressive type of cancer with limited therapeutic options. However, this type of cancer cells has shown overexpression of folate receptors, which bind with folic acid (FA) with high affinity. This feature can be used for targeting of nanocarriers, such as liposomes. In order to further examine the potential of increased efficacy by targeting the folate receptor, we prepared folate conjugated liposomes (DSPC/Chol/PEG/DSPE-

PEG-FA) and loaded them with doxorubicin (DOX), an anticancer drug. For this, we first synthesized and verified the conjugate between FA and PEG-lipid (FA-PEG-lipid). After that, liposomes were prepared with thin film hydration method followed by probe sonication. Three different types of targeted liposomes having different concentrations of FA-PEG-lipid in their membranes $(0.1$ $\mathrm{mol} \%, 0.5 \mathrm{~mol} \%$, and $1 \mathrm{~mol} \%$ ) were evaluated for their cytotoxicity (DOX-loaded liposomes) on MDA-MB-231 (epithelial human breast cancer cells), 4T1 (murine mammary carcinoma cells), and MCF7 (Human breast cancer cells); the two first are TNBC cells and overexpress FA receptor, the third does not. Cytotoxicity results proved that increasing amounts of FA on the surface of liposomes results in enhanced antitumor activity of liposomal-DOX in the case of the cancer cells, which overexpress the FA receptor.
\end{abstract}

Keywords: liposomes; folic acid; doxorubicin; breast cancer; TNBC

\section{Introduction}

Breast cancer (BC) is the most common malignancy and leading cause of cancer-related mortality among women worldwide [1,2]. Triple-negative breast cancer (TNBC) owes 15-20\% of all the invasive subtypes of breast cancer [3] and is characterized by absence of expression of estrogen receptors (ER), progesterone receptors (PR), and human epidermal growth factor receptor 2 (HER-2) on tumors cell membrane. This feature eliminates the benefits of endocrine therapy and treatment, therefore mainly relies on chemotherapy [4]. Even systemic chemotherapy with clinically approved drugs reflects poor response and high toxicity and develops multidrug resistance. However, this type of cancer cells has shown overexpression of folate receptors, which bind with folic acid (FA) with high affinity. Folate receptor (FR)-a is a glycosyl phosphatidylinositol-anchored membrane protein that is selectively overexpressed in $>90 \%$ of ovarian carcinomas [5], and to various extents in other epithelial cancers, but is only minimally distributed in normal tissues $[5,6]$. Therefore, liposomes conjugated to the folate ligand via a polyethylene glycol (PEG) spacer have been used to deliver chemotherapeutic agents, oligonucleotides, and markers to FR-bearing tumor cells. 
Doxorubicin (DOX), an anthracycline antibiotic, is one of the most commonly used anticancer agents for the treatment of various types of cancers, including breast cancer. It intercalates between base pairs of the DNA helix, thereby preventing DNA replication and ultimately inhibiting protein synthesis. Herein, we used DOX as an anticancer drug, to examine the potential of increased efficacy of DOX-liposomes by targeting the folate receptor. For this, we first synthesized and verified the conjugate between FA and PEG-lipid (FA-PEG-lipid). After that, we prepared liposomes with three different concentrations of FA-PEG-lipid in their membranes, loaded them with DOX, and compared their toxicity.

\section{Experiments}

\subsection{Materials}

Folic acid and N,N'-dicyclohexylcarbodiimide (DCC) were purchased from Sigma-Aldrich. Moreover, 1,2-Distearoyl-sn-glycerol-3-phosphatidyl-choline (DSPC), and 1,2-Distearoyl-sn-glycerol-3-phosphatidyl-ethanolamine- $N$-(methoxy(polyethylene-glyc ol)-2000) (PEG2000) were purchased from Lipoid, Germany. Cholesterol (Chol) was purchased from Sigma-Aldrich (Darmstadt, Germany). DSPE-PEG2000- $\mathrm{NH}_{2}$ was purchased from Avanti Polar Lipids. Doxorubicin, hydrochloric salt (DOX) was purchased by Tocris Bioscience, UK. Fetal Calf Serum (FCS) was obtained from Sigma (Darmstadt, Germany).

A bath sonicator (Branson) and a microtip-probe sonicator (Sonics and Materials, Leicestershire, UK) were used for liposome preparation. Protein concentrations were measured by Bradford microassay. The Cell Viability MTT assay was carried out with 3-(4,5-Dimethylthiazol-2-yl)-2,5-diphenyltetrazolium bromide (MTT) reagent (purchased by Sigma-Aldrich). All other reagents and chemicals used were of analytical grade and were purchased from Sigma-Aldrich.

\subsection{Methods}

\subsubsection{Synthesis of DSPE-PEG2000-FA Lipid (Folate-PEG-DSPE; FA-PEG-Lipid)}

Briefly, $12.65 \mathrm{mg}(0.028 \mathrm{mmol})$ of folic acid was dissolved in $1.2 \mathrm{~mL}$ DMSO and 0.208 $\mathrm{mL}(2.58 \mathrm{mmol})$ of pyridine. Next, $40 \mathrm{mg}(0.014 \mathrm{mmol})$ of DSPE-PEG2000-NH2, and 13 $\mathrm{mg}(0.063 \mathrm{mmol})$ DCC dissolved in $0.19 \mathrm{~mL}$ DMSO, were subsequently added. The reaction mixture was stirred overnight with a magnetic stirrer at room temperature (the progress of the reaction was monitored by TLC analysis). When the reaction was completed, $11 \mathrm{~mL}$ of water was added to the reaction mixture and the insoluble materials were removed by centrifugation at $15,000 \times \mathrm{g}$ for $15 \mathrm{~min}$. The supernatant was dialyzed 2 times in $50 \mathrm{mM} \mathrm{NaCl}$ for $24 \mathrm{~h}$ and then against water for $24 \mathrm{~h}$. The dialyzed product (folate-PEG-DSPE) was lyophilized and stored at $-20{ }^{\circ} \mathrm{C}$. The formation of folate-PEG-DSPE was confirmed by reverse-phase high-pressure liquid chromatography and by ${ }^{1} \mathrm{H}-\mathrm{NMR}$.

\subsubsection{Liposome Preparation and DOX Loading}

Liposomes of the following compositions were used in this study: (a) folate-PEG-liposomes (FA-LIP) composed of DSPC/cholesterol/DSPE-PEG2000-OMe/ folate-PEG-DSPE (62.6:31.3:6.0:0.1), (b) folate-PEG-liposomes composed of DSPC/ cholesterol/DSPE-PEG2000-OMe/folate-PEG-DSPE (62.3:31.2:6.0:0.5), (c) folate-PEGliposomes composed of DSPC/cholesterol/DSPE-PEG2000-OMe/folate-PEG-DSPE (62.0:31.0:6.0:1.0), and (d) control liposome not targeted with folic acid, composed of DSPC/cholesterol/DSPE-PEG2000-OMe (62.7:31.3:6.0). All liposome types were prepared by the thin-film-hydration method followed by probe sonication for size reduction. The resulting lipid films were hydrated at $60{ }^{\circ} \mathrm{C}$ with ammonium sulfate solution $(120 \mathrm{mM}$, $\mathrm{pH}$ 5.5, $300 \mathrm{mOsm})$, for DOX loading. For physicochemical characterization, liposomes were hydrated with PBS buffer ( $\mathrm{pH}$ 7.40, $300 \mathrm{mOsm}$ ). Vesicle size was reduced by subjection to a high intensity (750 Watt) probe sonicator (Sonics and Materials, Leicester- 
shire, UK) for $15 \mathrm{~min}$ and then left at $60^{\circ} \mathrm{C}$ for $1 \mathrm{~h}$, to anneal any structural defects. DOX was loaded in LIP and FA-LIP by the active loading method, as described in detail elsewhere [7]. In brief, the vesicles formulated in ammonium sulfate $\left(\left[\mathrm{NH}_{4}\right]_{2} \mathrm{SO}_{4}(120\right.$ $\mathrm{mM})$ ) were resuspended in PBS, $\mathrm{pH}$ 7.4, for exchange of the dispersion media, and incubated (at a lipid concentration of $2.4 \mathrm{mg} / \mathrm{mL}$ ) with $0.0887 \mathrm{mg} / \mathrm{mL}$ DOX solution (in PBS) (corresponding to a lipid/DOX ratio equal to $27: 1(\mathrm{w} / \mathrm{w})$ ), for $60 \mathrm{~min}$ at $60^{\circ} \mathrm{C}$. Lipid content of liposomes was measured by the Stewart assay, a colorimetric method used routinely for the quantification of phospholipids [8]. Liposomes were purified from non-encapsulated DOX by repeated ultracentrifugation for $1 \mathrm{~h}$ (each) at 60,000 rpm (Sorvall WX90 Ultra, Thermo Scientific, Waltham, MA, USA).

\subsubsection{Physicochemical Properties of Liposomes}

Liposomes were characterized for their lipid concentration, mean diameter, size distribution, and zeta-potential. Vesicle size distribution was measured by dynamic light scattering (Malvern Instruments, Zetasizer Nano SZ, Malvern, UK). Sizes reported correspond to the z-average means of the hydrodynamic diameters of the liposomes. For $\zeta$-potential values, the electrophoretic mobility of the liposome dispersions was measured at $25{ }^{\circ} \mathrm{C}$, by the same instrument. Zeta potential values were obtained (by the instrument) from the electrophoretic mobility, according to the Smoluchowski equation.

The final D/L (drug/lipid) ratio (w/w) was compared with the initial one, for calculation of the DOX encapsulation efficiency (\%) of each liposome type, according to Equation (1):

$$
\mathrm{EE}(\%)=\frac{D / L_{\text {final }}}{D / L_{\text {initial }}} \times 100
$$

\subsubsection{Cell Culture Studies}

Four cell lines were used in this study: (i) MDA-MB-231 (epithelial human breast cancer cells), (ii) 4T1 (murine mammary carcinoma cells), (iii) MCF7 (human breast cancer cells), and (iv) HEK (human HEK-293 embryonic kidney cells). Cancel cells were grown in RPMI 1640 medium supplemented with 10\% FBS and 1\% antibiotic-antimycotic and the normal cells were grown in DMEM medium supplemented with 10\% FBS and $1 \%$ antibiotic-antimycotic solution (Invitrogen, Carlsbad, CA, USA). The cells were cultured at $37^{\circ} \mathrm{C}, 5 \% \mathrm{CO}_{2}$ /saturated humidity. Medium was changed every $2-3$ days.

The toxicity of the various types of liposomes towards cancer and normal cells was evaluated by the corresponding reduction in cell viability after $24 \mathrm{~h}$ incubation with test (DOX-FA-LIP) or control formulation (DOX-LIP), by the MTT assay. For each experiment, cells were seeded overnight at $37^{\circ} \mathrm{C}$ at a density of $5 \times 10^{4}$ cells per well, in 24-well plates and then incubated for $24 \mathrm{~h}$ at $37{ }^{\circ} \mathrm{C}\left(5 \% \mathrm{CO}_{2} /\right.$ saturated humidity $)$ with $0.4 \mathrm{~mL}$ growth medium and $0.1 \mathrm{~mL}$ of the formulation evaluated in each case. Cell viability (\%) was calculated by Equation (2):

$$
\text { Viability }(\%)=\frac{O D-570_{\text {sample }}-O D-570_{\text {background }}}{O D-570_{\text {control }}-O D-570_{\text {background }}} \times 100 \text {, }
$$

where OD-570 control corresponds to untreated cells (or PBS control) and OD-570 background corresponds to MTT without cells. All samples were diluted in order to achieve DOX concentrations of $3 \mu \mathrm{M}$.

\section{Results}

\subsection{Physicochemical Properties and Stability of FA-LIP and LIP}

All liposome dispersions were characterized for their size distribution (mean hydrodynamic diameter and polydispersity index) and their zeta-potential (Table 1). It should be pointed out that the liposomes were formulated in PBS buffer, $\mathrm{pH}$ 7.40. 
Table 1. Physicochemical properties of Folic acid-Liposomes FA-LIP and LIP, formulated in phosphate buffer saline PBS buffer ( $\mathrm{pH} 7.40)$.

\begin{tabular}{ccccc}
\hline DSPE-PEG2000-FA (Mole\%) & Mean Hydrodynamic Diameter (nm) & PDI & $\zeta$-Potential (mV) & DOX Loading (\%) \\
\hline 0 & $99.7 \pm 3.05$ & 0.243 & $-5.49 \pm 0.33$ & $91.4 \pm 2.2$ \\
0.1 & $99.9 \pm 1.4$ & 0.159 & $-4.38 \pm 0.91$ & $94.1 \pm 1.7$ \\
0.5 & $124.1 \pm 0.7$ & 0.192 & $-7.4 \pm 1.6$ & $95.9 \pm 2.2$ \\
1 & $126.5 \pm 2.4$ & 0.161 & $-5.7 \pm 1.1$ & $97.3 \pm 2.3$ \\
\hline
\end{tabular}

As seen in Table 1, the mean hydrodynamic diameter of all the liposomes prepared was in the nano-range, between 99 and $127 \mathrm{~nm}$. The polydispersity index values were between 0.159 and 0.243 . The addition of FA-PEG-lipid was shown to increase slightly the mean diameter of the vesicle, and more precisely, a higher molar ratio of the lipid incorporated is connected with higher diameter. Zeta potential values were in the range between -4.38 and $-7.41 \mathrm{mV}$.

Additionally, the loading of DOX achieved in all liposome types ranged between $91 \%$ and $97 \%$. The addition of FA had no significant effect on DOX loading, in comparison with the untargeted liposome.

\subsection{In Vitro Anticancer Activity}

As seen in Figure 1, incubation of HEK (Figure 1A) and MCF-7 cells (Figure 1B), which do not overexpress the FA receptor, with targeted DOX liposomes (at the same DOX concentration), does not seem to have any effect to enhance the anticancer activity of the non-targeted DOX-liposomes (control), as anticipated. Oppositely, the targeted liposomes seem to enhance the anticancer activity of DOX, compared to the control liposomes, in the cases of cells, which overexpress the FA receptor, such as the 4T1 cells (Figure 1C) and the MDA-MB-231 cells (Figure 1D). The later effect is seen only when the FA density on the targeted liposomes is higher than $0.5 \%$ and especially in the case of the liposomes that have $1 \mathrm{~mol} \%$ of FA-conjugated PEG-lipid in their membranes.

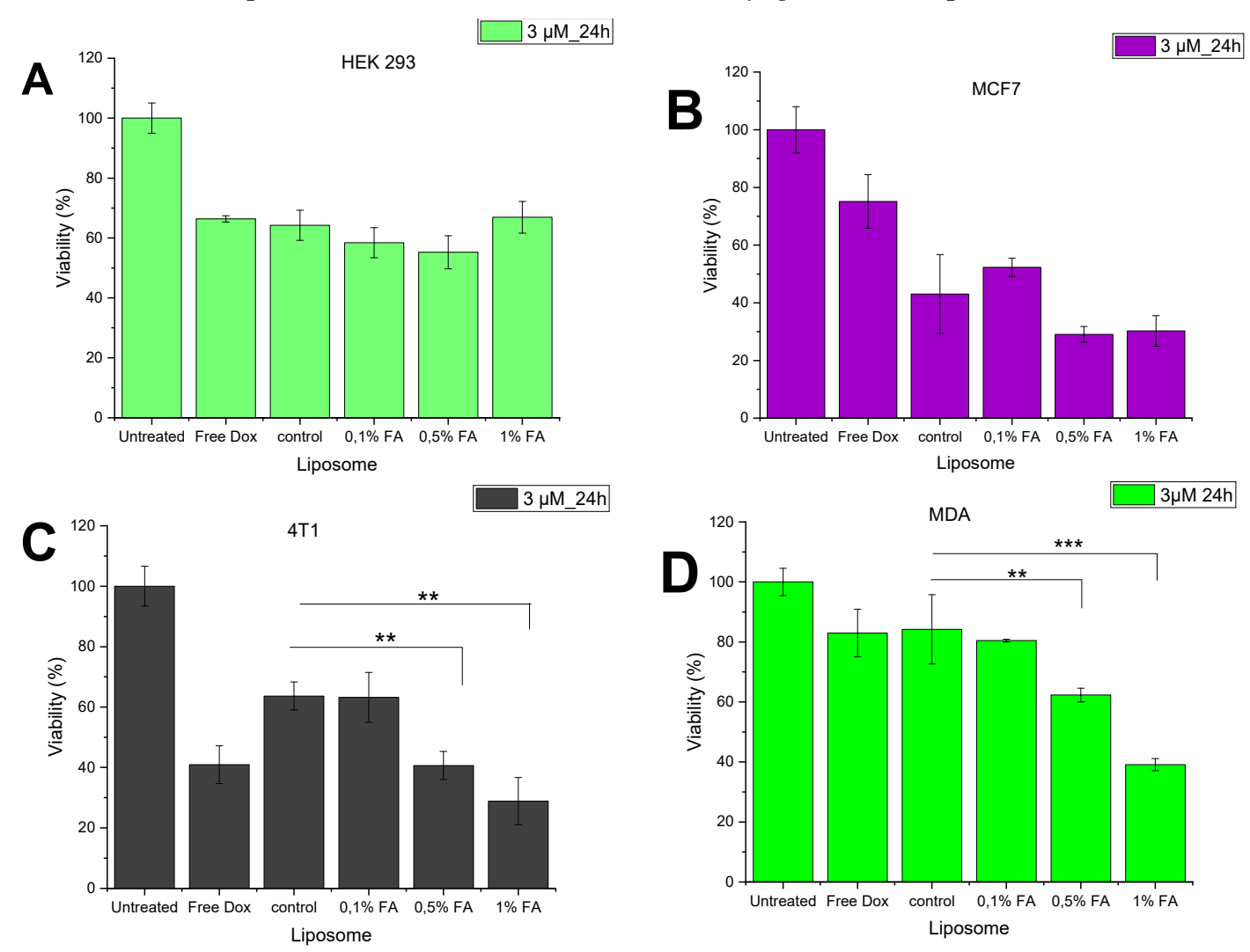

Figure 1. Viability of cells after incubation with folic acid (FA)-targeted Doxorubicin-Liposomes (DOX-LIP) and control (non-targeted) liposomes and free doxorubicin (DOX), corresponding to a DOX concentration of $3 \mu \mathrm{M}$ for $24 \mathrm{~h}$ (A) HEK cells, (B) MCF-7 cells, (C) 4 T1 cells, and (D) MDA-MB-231 cells. ${ }^{* *}$ and ${ }^{* * *}$ correspond to $p<0.01$ and $p<0.001$, respectively. 


\section{Discussion}

Herein, we investigated the potential to target DSPE-Chol-PEG liposomes with FA and its effect on the anticancer properties of DOX. Furthermore, we evaluated the ability to load FA-LIP with DOX using the active loading method, in order to develop potential improved anticancer chemotherapeutic systems. All formulations of FA-LIP as well as LIP (which were prepared as control vesicles), had mean diameters in the nano-range, between 99 and $127 \mathrm{~nm}$ (Table 1), polydispersity values below 0.250, and slightly negative zeta potential values. Concerning the capability of using the active loading protocol to load DOX into FA-LIP, the current results confirmed that high amounts of DOX can be loaded into FA-LIP successfully.

The in vitro experiments carried out with DOX-FA-LIP on HEK, MDA-MB-231, MCF7, and 4T1 cells demonstrated interesting anticancer potential of DOX-FA-LIP with 1 mol\% of FA-PEG-lipid in their membranes, toward the cancel cell types that overexpress FA receptor (Figure 1).

Preliminary FITC-uptake studies indicate that the targeting potential of the current liposomes is low. Perhaps this is linked to the fact that FA was attached on lipid-PEG conjugates with PEG2000 molecules; the use of higher MW PEG to better expose FA on the liposome surface has been proposed by others, as a method for enhancing targeting potential. Currently, we are evaluating the effect of the PEG MW on the targeting potential of FA-targeted liposomes.

\section{Conclusions}

In this study, folic acid-targeted DSPE-PEG2000 lipid (DSPE-PEG-FA) was successfully synthesized and utilized as a homing ligand to target DOX to cancer cells, which overexpress the FA receptor. Our data show that liposomes with DSPE-PEG-FA can efficiently load DOX and form stable nano assemblies with a size within the nanoscale. Folic acid as a ligand on liposome surface can improve the antitumor activity of liposomal DOX towards triple-negative breast cancer (TNBC) cells; we are currently evaluating methods to further optimize the targeted DOX-liposomes.

Author Contributions: S.G.A. conceived the experiments and got the funding. S.M. designed the synthesis of DSPE-PEG-FA lipid and the molar composition of each liposome. A.M. conceived the experiments regarding the cells. F.G. designed and helped with the execution of the cell experiments. E.L. performed all the experiments needed together with M.M.; E.L. wrote a first draft of the paper. E.L., F.G., and M.M. gathered and analyzed the data. S.A. corrected the draft paper and wrote the final version. All authors have read and agreed to the published version of the manuscript.

Institutional Review Board Statement: Not applicable.

Informed Consent Statement: Not applicable.

Data Availability Statement: The data presented in this study are available in the results section of the article.

Acknowledgments: This research has been co-financed by the European Regional Development Fund of the European Union and Greek national funds through the Operational Program Competitiveness, Entrepreneurship and Innovation, under the call RESEARCH-CREATE-INNOVATE (project code: MIS5031802).

Conflicts of Interest: The authors declare no conflicts of interest. The founding sponsors had no role in the design of the study; in the collection, analyses, or interpretation of data; in the writing of the manuscript; and in the decision to publish the results.

\section{References}

1. EER Preliminary Cancer Incidence Rate Estimates for 2017, and Diagnosis Years 2000 to 2017, SEER 18. Available online: https://seer.cancer.gov/statistics/preliminary-estimates/ (accessed on April 2020).

2. Siegel, R.L.; Miller, K.D.; Jemal, A. Cancer statistics, 2017. CA Cancer J. Clin. 2017, 67, 7-30, doi:10.3322/caac.21387. 
3. He, Y.; Jiang, Z.; Chen, C.; Wang, X. Classification of triple-negative breast cancers based on Immunogenomic profiling. J. Exp. Clin. Cancer Res. 2018, 37, 327, doi:10.1186/s13046-018-1002-1.

4. Gangi, A.; Chung, A.; Mirocha, J.; Liou, D.Z.; Leong, T.; Giuliano, A.E. Breast-conserving therapy for triple-negative breast cancer. JAMA Surg. 2014, 149, 252-258, doi:10.1001/jamasurg.2013.3037.

5. Weitman, S.D.; Lark, R.H.; Coney, L.R.; Fort, D.; Frasca, V.; Zurawski, V.; Kamen, B. Distribution of the folate receptor GP38 in normal and malignant cell lines and tissue. Cancer Res. 1992, 52, 3396-3401.

6. Elnakat, H.; Rantnam, M. Distribution, functionality and gene regulation of folate receptor isoforms: Implications in targeted therapy. Adv. Drug Deliv. Rev. 2004, 56, 1067-1084.

7. Zucker, D.; Marcus, D.; Barenholz, Y.; Goldblum, A. Liposome drugs' loading efficiency: A working model based on loading conditions and drug's physicochemical properties. J. Control. Release 2009, 139, 73-80.

8. Stewart, J.C.M. Colorimetric determination of phospholipids with ammonium ferrothiocyanate. Anal. Biochem. 1980, 104, 1014. 